\title{
A Comparison of Optimal and Suboptimal Processors for Classification of Buried Metal Objects
}

\author{
Ping Gao, Student Member, IEEE, and Leslie Collins, Member, IEEE
}

\begin{abstract}
Classification of metal objects is important for landmine and unexploded ordnance applications. Previously, we have investigated optimal classification of landmine-like metal objects using wideband frequency-domain electromagnetic induction data [1]. Here, a suboptimal processor, which is computationally less burdensome than the optimal processor, is discussed. The data is first normalized, exploiting the fact that the level of the response changes significantly while the structure of the magnitude of the response changes only slightly as the target/sensor orientation changes for the class of objects considered. Results indicate that the suboptimal processor performance approaches that of the optimal classifier on normalized data. Thus, normalization mitigates the uncertainty resulting from the target/sensor orientation.
\end{abstract}

Index Terms - Bayes procedures, data processing, electromagnetic induction, signal classification.

\section{INTRODUCTION}

$\mathbf{T}$ IME-DOMAIN electromagnetic induction (EMI) sensors have been used extensively for landmine and unexploded ordnance (UXO) detection. In order to discriminate targets of interest from other pieces of metal, several modifications to traditional EMI sensors have been considered [2]-[6]. One promising approach is to operate the EMI sensor in the frequency-domain utilizing wideband excitation. The frequency dependence of the induced fields excited by buried conducting targets can then be exploited by a detector.

We previously considered classification of various metal objects using wideband frequency-domain EMI data [1]. The development of the optimal processor integrates a numerical model of wideband EMI responses [2] and a Bayesian decision-theoretic approach. It is of importance in this approach to accurately model the wideband EMI response and to consider uncertainties regarding the target/sensor orientation. We included appropriate treatment of such uncertainty, resulting in significant performance improvement over algorithms in which such information was ignored.

The drawback to the optimal processor is the computational burden. In some scenarios, such as UXO detection and classification where data processing can be performed offline, this computational complexity is not an issue. However, in hu-

Manuscript received March 4, 1999. This work was sponsored by the Army Research Office under Grant DAAH04-96-1-448 (Demining MURI). The associate editor coordinating the review of this manuscript and approving it for publication was Dr. Y. Hua.

The authors are with the Electrical and Computer Engineering Department, Duke University, Durham, NC 27708-0291 USA (e-mail: lcollins@ee.duke.edu).

Publisher Item Identifier S 1070-9908(99)05791-0. manitarian and military demining, real-time decision making is required, thus computational complexity becomes problematic.

Therefore, the goal of this work is to investigate suboptimal classifiers that continue to incorporate the physical nature of the wideband frequency-domain EMI signal, but are less computationally burdensome. It is shown on both simulated and experimental data that by normalizing the sensor output, a suboptimal processor operating on the normalized data can achieve nearly the same, or in some cases, better classification performance than that of the optimal processor operating on unnormalized data.

\section{APPROACH}

A model-based Bayesian decision-theoretic approach was investigated to discriminate man-made metal targets under conditions where the target/sensor orientation is unknown, since the exact sensor position (where measurements are obtained) relative to the underground objects is unknown in practice [1]. When the exact dimensions, constitutive parameters of the target, and the horizontal and vertical distance from the center of the sensor to that of the target are specified, the theoretical wideband EMI response can be calculated. In order to model the wideband EMI signature of these targets, a method of moment (MoM) analysis was used to predict the theoretical response (in-phase and quadrature voltages) from the targets at the frequencies of interest. To test the effectiveness of the approach, data was collected from a prototype wideband frequency-domain EMI sensor, the GEM-3 [7], developed by Geophex, Ltd. The discrimination algorithm was applied to both simulated data and experimental data measured using the sensor.

Five metal targets were used for the simulations: an aluminum cone, an aluminum barbell, an aluminum disk, a thick brass disk, and a thin brass disk. This target set was selected because of the similarities in shape of three of the targets to antitank mines and two of the targets to clutter. Twenty-one frequencies, ranging from 4 to $24 \mathrm{kHz}$ in a $1-\mathrm{kHz}$ spacing, were used for both simulations and measurements. It is assumed that the sensor is subject to a small amount of additive Gaussian noise, which was verified during the experimental data acquisition. Let $H_{i}$ represent the hypothesis that the $i$ th target is present, where $i=1, \cdots, K$. The received data from the $i$ th target can be expressed as $r_{i j}=A_{i j}+n_{j}$, where $j$ corresponds to frequency, $r_{i j}$ is the received data of the $i$ th target from the sensor, $A_{i j}$ is the model prediction for the $i$ th target at the $j$ th frequency when the target is at a known position, and $n_{j}$ is Gaussian noise with zero mean and variance of $\sigma_{n_{j}}^{2}$. Let $q_{i}$ represent the a priori probability that 
hypothesis $H_{i}$ is true, and assume that the cost of a correct decision is zero, and the cost of a wrong decision equals one. The optimal solution for this classification problem [8] is to decide that $H_{i}$ is true if

$$
\frac{p\left(H_{i} \mid r\right)}{p\left(H_{k} \mid r\right)}=\frac{q_{i} p\left(r \mid H_{i}\right)}{q_{k} p\left(r \mid H_{k}\right)}>1
$$

is satisfied for any $k \neq i$, where $p\left(r \mid H_{i}\right)$ is the probability density or likelihood function of data $r$ given $H_{i}, p\left(H_{i} \mid r\right)$ is the a posteriori distribution or discriminant function [9], and $r$ is the in-phase and quadrature (assumed independent) data from the sensor at a known position. A uniform prior on $q_{i}$ is assumed, therefore, $q_{i}=1 / K$. When data $r$ is received, we decide in favor of hypothesis $H_{i}$ where $q_{i} p\left(r \mid H_{i}\right)=$ $\max _{k}\left\{q_{k} p\left(r \mid H_{k}\right)\right\}, k=1, \cdots, K$. Thus, we decide in favor of a hypothesis that has the largest a posteriori probability among the $K$ probability density functions. After simplifying, the alternative discriminant function [9] is

$$
\log p^{\prime}\left(r \mid H_{i}\right)=-\left(r-A_{i}\right)^{T} \Sigma^{-1}\left(r-A_{i}\right)
$$

where $A_{i}$ is the model prediction of the response of the $i$ th target, and $\Sigma$ is the covariance matrix of $r$.

The discriminant function in (2) is optimal only when a target is at a known position, when all the parameters are known, and when the sensor is subject to Gaussian noise. It can be implemented as a bank of "matched" filters. The noise is not identically distributed, and the signals are not of equal energy; thus, these two facts result in a formulation which is similar to, but not identical to, the traditional matched filter $r^{T} A_{i}$.

A more accurate assumption in practice is that the height and horizontal position between the center of the target and that of the sensor are uncertain. In this case, the matched filterbank is not the optimal solution. The optimal alternative discriminant is

$$
p\left(r \mid H_{i}\right)=\iiint p\left(r \mid H_{i}, h, x, y\right) p(h) p(x, y) d h d x d y
$$

where $h$ represents the height of the sensor from the target; $x, y$ represent the horizontal position of the center of the target relative to the sensor; and $p(h)$ and $p(x, y)$ are the a priori distributions of the position variables, which are assumed independent.

Four scenarios were considered in simulations:

1) target at a known, fixed position;

2) $h \sim N\left(20 \mathrm{~cm}, 1.532 \mathrm{~cm}^{2}\right)$, and $x, y=0$;

3) $x, y$ is uniformly distributed in a $20 \times 20 \mathrm{~cm}$ square, and $h$ is fixed;

4) both height and horizontal position are uncertain, following the same distributions as above.

The priors placed on the uncertain positional parameters are consistent with those observed in demining applications [10].

For each target, measurements were taken at 164 positions using the GEM-3. The choices of these locations follow a Gaussian distribution for height and a uniform distribution for horizontal position as in the simulations.

\section{Alternative Processor Design}

The wideband EMI responses for the same target at different heights and horizontal positions show a somewhat "parallel" structure as a function of frequency [11]. The overall level varies substantially as the target/sensor orientation changes, but the basic structure of the response as a function of frequency changes only slightly. This is because the transfer function of a nonferrous target can be expressed as $H(\omega)=$ $\Sigma_{n}\left[a_{n} \omega\left[\omega-j \omega_{n}\right]\right.$, where $\omega$ is radian frequency, $\omega_{n}$ is the $n$th resonant frequency, and $a_{n}$ is the coefficient corresponding to each resonant frequency. When the target aspect ratios (length versus diameter) are approximately equal, and only the first mode dominates, the frequency response scales directly with variable orientations [11]. Based on this phenomenon, we hypothesized that if the wideband EMI signatures were normalized, performance could be evaluated using a processor that avoids calculating the integration over position uncertainty [as in (3)]. Then, the processor can be implemented as previously described for the "matched filter" case after normalization. Therefore, the outputs of the sensor are normalized so that the energy in the response is equal to unity. This operation essentially decreases the uncertainties of the data collected in an uncertain environment. The suboptimal processor takes the form of (2), which uses the mean signature after normalization over all uncertainties as $A_{i}$ for the $i$ th target.

The performance of a matched-filter-like processor cannot exceed the performance of an optimal classifier operating on the same data set. However, by using the suboptimal processor, computational savings are proportional to that required to evaluate the integral shown in (3), thus can be implemented in real time.

\section{RESULTS}

Table I illustrates the performance of the various processors operating on simulated data before and after normalization for the five metal objects. For the fixed position case, the matchedfilter-like processor is optimal. The results indicate that when there is no uncertainty in the target/sensor orientation (fixed position), performing the normalization degrades the performance of the processor. However, when the target positions are uncertain, better performance can be achieved if the output is normalized. Also, the matched filter performance is quite close to the optimal classifier performance for the normalized data. This indicates that applying the sub-optimal processor to normalized data only slightly sacrifices performance, but computational time is reduced significantly. Interestingly, the optimal classifier operating on the normalized data outperforms the optimal classifier operating on the unnormalized data. This occurs because the normalization uncorrelates the signals to a small extent, which improves classification performance [12]. For instance, the average correlation coefficient decrease before and after normalization between the target 3 and 5 is $0.711 \times 10^{-4}$.

Table II illustrates the performance of various processors using measured data. Data was gathered from four out of five targets used in simulations. The suboptimal processor using normalized data also performs better in general than the processor using raw sensor output, and the optimal processor after normalization achieves better performance than the processor without normalization. This performance improvement validates the hypothesis that by normalizing the sensor data, the uncertainties associated with the sensor/target position can 
TABLE I

Performance of the Various Processors Before and After Normalization Using Simulated Data When 1) Target Position Is Known and Fixed, 2 ) $h \sim N\left(20 \mathrm{~cm}, 1.532 \mathrm{~cm}^{2}\right)$ and $x, y$ Is Fixed, 3) Horizontal Position Is UNIFORMLy Distributed in a $20 \mathrm{~cm} \times 20 \mathrm{~cm}$ SQUARE AND Height Is FiXed, AND 4) Both Height and Horizontal Position Are Random, Following the Distributions Mentioned Above. "No Norm": Processing Occurred Without Normalization. "Norm": Processing Followed Normalization. "Opt": Optimal Classifier. "MF": Matched-Filter-Like Processor

\begin{tabular}{|c|c|c|c|c|c|c|c|}
\hline & \multicolumn{5}{|c|}{ Probability of Correct Classification } \\
\hline & & & Target 1 & Target 2 & Target 3 & Target 4 & Target 5 \\
\hline \multirow{2}{*}{$\begin{array}{l}\text { Fixed } \\
\text { Position }\end{array}$} & No Norm. & MF/Opt. & 1 & 1 & 1 & 1 & 1 \\
\hline & Norm. & MF/Opt. & 1 & .976 & 1 & .976 & 1 \\
\hline \multirow{4}{*}{$\begin{array}{l}\text { Height } \\
\text { Uncertain }\end{array}$} & \multirow{2}{*}{ No Norm. } & MF & .832 & .351 & .518 & .081 & .620 \\
\hline & & Opt. & .999 & .857 & .997 & .830 & 1 \\
\hline & \multirow{2}{*}{ Norm. } & MF & 1 & .973 & 1 & .972 & 1 \\
\hline & & Opt. & 1 & .977 & 1 & .978 & 1 \\
\hline \multirow{4}{*}{$\begin{array}{l}\text { Horizontal } \\
\text { Position } \\
\text { Uncertain }\end{array}$} & \multirow{2}{*}{ No Norm. } & MF & .804 & .411 & .573 & .085 & .655 \\
\hline & & Opt. & 1 & .828 & 1 & .810 & .999 \\
\hline & \multirow{2}{*}{ Norm. } & $\mathrm{MF}$ & 1 & .849 & 1 & .754 & 1 \\
\hline & & Opt. & 1 & .866 & 1 & .810 & 1 \\
\hline \multirow{4}{*}{$\begin{array}{l}\text { Both } \\
\text { Height \& } \\
\text { Horiz. } \\
\text { Position } \\
\text { Uncertain }\end{array}$} & \multirow{2}{*}{ No Norm. } & MF & .758 & .340 & .506 & .073 & .492 \\
\hline & & Opt. & .994 & .701 & .990 & .700 & .996 \\
\hline & \multirow{2}{*}{ Norm. } & MF & 1 & .848 & 1 & .759 & 1 \\
\hline & & Opt. & 1 & .879 & 1 & .823 & 1 \\
\hline
\end{tabular}

TABLE II

Performance of the Suboptimal and Optimal Processors Before and After Normalization Evaluated on Measured Data. "MF": Matched-Filter-Like or Suboptimal Processor. "No Norm": Processing Occurred Without Normalization. "Opt Proc": Optimal Processor. "Norm": Processing Followed Normalization

\begin{tabular}{l|c|c|c|c}
\hline & Target 2 & Target 3 & Target 4 & Target 5 \\
\hline Opt. Proc., No Norm. & 0.91 & 0.77 & 0.69 & 0.81 \\
\hline Opt. Proc., Norm. & 0 & 0.98 & 1 & 0.99 \\
\hline MF, No Norm. & 0.23 & 0.24 & 0.03 & 0.14 \\
\hline MF, Norm. & 1 & 0.99 & 0.89 & 0.83 \\
\hline
\end{tabular}

be decreased. Also, the performance of a matched-filter-like processor operating on normalized data is very close to that of the optimal processor operating on normalized data. However, the computational load is reduced significantly.

\section{DISCUSSION}

Using wideband frequency-domain EMI data, metal objects of different dimensions and materials can be discriminated via classification algorithms carefully designed using signal detection theory. Since the uncertainty inherent in the sensor output is not only due to the additive noise but also the unknown target/sensor orientation, both facts should be considered during the development of the processor. The optimal classifier, which integrates over the uncertainty in the sensor/target orientation, can provide dramatic performance improvement over a matched-filter-like processor; however, it suffers from a computational burden and is not always practical. For the class of targets considered (target aspect ratio close to one), normalizing the wideband EMI responses, which exploits the physical nature of the signals, mitigates the uncertainties of the response due to the unknown target position. By performing the normalization, a suboptimal processor can provide significant performance improvement over suboptimal processors that operate on raw sensor output, and computational time can be dramatically decreased. Since for general targets the frequency-domain EMI response does not simply scale while the target/sensor orientation changes, we will investigate the relationship of the longitudinal and azimuthal modes of the response and integrate this information into the classifier design.

\section{ACKNOWLEDGMENT}

The authors would like to thank N. Geng, L. Carin, L. Nolte, S. Tantum, D. Keiswetter, I. J. Won, and R. Weaver for their generous help regarding this work.

\section{REFERENCES}

[1] P. Gao et al., "Classification of landmine-like metal targets using wideband electromagnetic induction," submitted for publication.

[2] N. Geng et al., "Wideband electromagnetic induction for metal-target identification: theory, measurement and signal processing," Tech. Rep., Duke Univ., Durham, NC, Sept. 1997.

[3] L. Collins, P. Gao, and L. Carin, "An improved Bayesian decision theoretic approach for land mine detection," IEEE Trans. Geosci. Remote Sensing, vol. 37, pp. 811-819, Mar. 1998.

[4] C. E. Baum, Low frequency near-field magnetic scattering from highly, but not perfectly conducting bodies," Phillips Lab. Interaction Note 499, Nov. 1993.

[5] G. D. Sower and S. P. Cave, "Detection and identification of mines from natural magnetic and electromagnetic resonances," Proc. SPIE, 1995, FL.

[6] S. Vitebskiy and L. Carin, "Late-time resonant frequencies of buried bodies of revolution," IEEE Trans. Antennas Propagat., vol. 44, pp. 1575-1583, 1996.

[7] I. J. Won, D. A. Keiswetter, and D. R. Hansen, "GEM-3: A monostatic broadband electromagnetic induction sensor," J. Environ. Eng. Geophys., vol. 2, pp. 53-64, Aug. 1997.

[8] J. C. Hancock and P. A. Wintz, Signal Detection Theory. New York: McGraw Hill, 1966, pp. 80-84.

[9] R. J. Schalkoff, Pattern Recognition: Statistical, Structural and Neural Approaches. New York: Wiley, 1992, pp. 34-43.

[10] "Mine countermine operations," Army FM 20-32, May 1998.

[11] L. Carin, Wideband time- and frequency-domain EMI: phenomenology and signal processing," Tech. Rep., U.S. Air Force Philip's Lab. Project, Oct. 1998.

[12] J. G. Rasimas, "Signal detection and classification of targets using multiple aspect angles," M.S. thesis, Duke Univ., Durham, NC, 1998. 